\title{
Angiosarcoma of the scalp diagnosed by the presence of neck inflammation: a case report
}

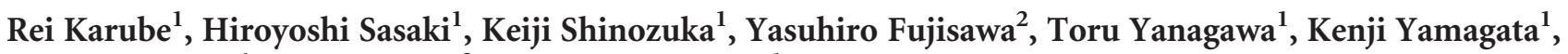 \\ Kojiro Onizawa ${ }^{1}$, Fujio Otsuka ${ }^{2}$ and Hiroki Bukawa ${ }^{1}$
}

Angiosarcoma is an uncommon malignancy, which spread out from the endothelial cells of vessels. Scalp angiosarcoma with cervical lymph node metastasis is particularly rare. This article describes a rare case of angiosarcoma of the scalp, presenting as neck inflammation. Imaging procedures such as computed tomography (CT), magnetic resonance image (MRI) and ultrasonography (US) were not sufficient to diagnose this case. A needle biopsy provided an effective and accurate diagnosis of cervical lymph node metastasis. Additional observation and physical examination was required to diagnose the origin of the primary cancerous lesion. Once the angiosarcoma diagnosis was confirmed histologically, sequential weekly and monthly docetaxel (DTX) treatment was effective in preventing reoccurrence. Nonetheless, the optimization of angiosarcoma treatment remains a future goal. Although patients generally describe pain and swelling at the primary lesion site, this patient complained only of painful neck inflammation, without any indication of pain or swelling of the scalp. A revised diagnostic protocol should note that cervical lymph node metastasis of unknown primary origin may result from angiosarcoma of the scalp.

International Journal of Oral Science (2012) 4, 166-169; doi:10.1038/ijos.2012.36; published online 15 June 2012

Keywords: angiosarcoma; docetaxel; neck inflammation; needle biopsy; scalp

\section{INTRODUCTION}

Angiosarcoma is a rare malignant tumor, accounting for only $1 \%-$ $5 \%$ of all soft tissue sarcomas. ${ }^{1-2}$ Cutaneous angiosarcoma is the most common presentation of this neoplasm, which affects the facial skin and scalp regions. ${ }^{3}$ Head and neck angiosarcoma is usually seen in patients older than 60 years of age, and slightly more often in males. ${ }^{1,3-4}$ The pathology usually follows an aggressive course, with progressive local invasion and early metastasis. ${ }^{3}$ We describe a case of angiosarcoma of the scalp, presenting as neck inflammation. A needle biopsy provided an effective and accurate diagnosis of cervical lymph node metastasis. Additional observation and physical examination was required to diagnose the origin of the primary cancerous lesion. Once the angiosarcoma diagnosis was confirmed histologically, sequential weekly and monthly docetaxel (DTX) treatment was effective in preventing reoccurrence. Nonetheless, the optimization of angiosarcoma treatment remains a future goal.

\section{CASE REPORT}

A 69-year-old man presented with swelling in the left cervical region that had persisted for 3 months. He had had a gastric ulcer, but no significant family history of cancer. A physical examination revealed a reddish, stiff swelling with tenderness from the left submandibular to the parotid-masseter region (Figure 1). An oral examination did not provide any diagnostically relevant details. The laboratory data at the first visit showed that white blood cell count and C-reactive protein were $6.0 \times 10^{3} \mu \mathrm{L}^{-1}$ and $0.09 \mathrm{mg} \cdot \mathrm{dL}^{-1}$, respectively, and the other laboratory data was also within normal limit, so the general inflammation was excluded. However, four symptoms of inflammation such

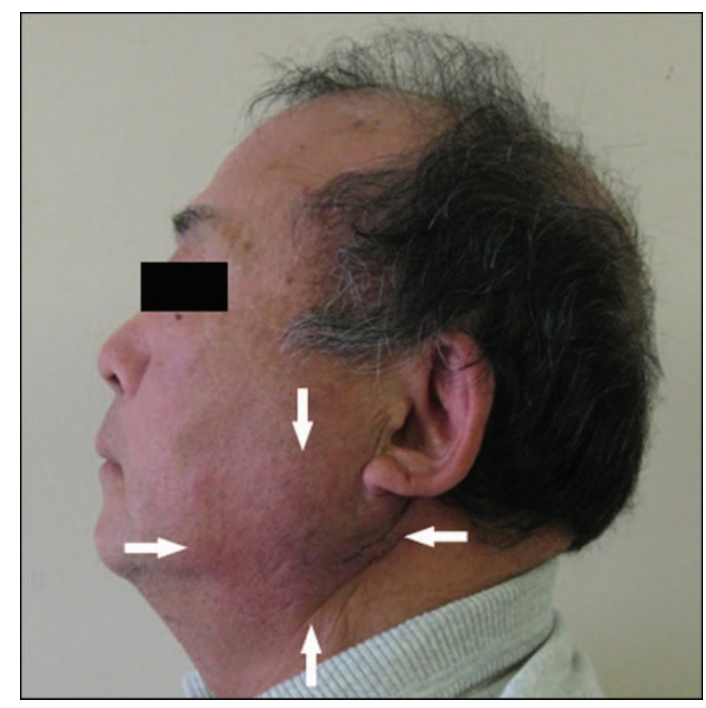

Figure 1 Pre-treatment view reveals a reddish swelling from the left submandibular to the parotid-masseter region (white arrows). 
as heat, redness, pain and swelling were observed in the left neck, indicating that mild local inflammation. A computed tomography (CT) scan showed that the subcutaneous legion $(25 \mathrm{~mm} \times 19 \mathrm{~mm} \times$ $10 \mathrm{~mm}$ ) was slightly swollen with an enhancement and expanded outward with a swollen, round, ring-enhanced lymph node in the left cervical region (Figure 2a). A magnetic resonance image (MRI) revealed a high-signal lesion on T2-weighted images under the left parotid gland (Figure 2b). An 18F-fluorodeoxyglucose positron emission tomography (PET) CT scan image showed focal uptake in the left cervical region (Figure $2 \mathrm{c}$ and $2 \mathrm{~d}$ ). Ultrasonography (US) showed a heterogeneous lesion in the cervical region, and gastrointestinal endoscopy revealed atrophic gastritis with no sign of a tumor (data not shown). These examinations indicated a cervical lymph node metastasis but did not detect the primary lesion.

An echo-guided needle biopsy of the neck mass was carried out to identify the primary tumor. Pathology results (hematoxylin and eosin staining) indicated atypical, enlarged pleomorphic endothelial cells and microcyst formation (Figure 3a). Immunohistochemistry revealed that the tumor cells were positive for CD31, a marker for immature endothelial cells (Figure 3b), and negative for factor VIIIrelated antigen, Keratin, S-100 and Desmin (data not shown). The pathological and immunohistochemical results pointed to a diagnosis of angiosarcoma. Although the patient only complained of a painful neck swelling, a detailed examination revealed that the primary tumor was angiosarcoma of the scalp. Although the patient experienced an itchy scalp, he was unaware of a $45 \mathrm{~mm} \times 30 \mathrm{~mm}$ crusty ulcer on the vertex of his head (Figure 4). Consulting dermatologists confirmed a final diagnosis of angiosarcoma of the scalp with left cervical lymph node metastasis (T1bN1M0, stage IV). The specialists in dermatology diagnosed the vertex lesion as angiosarcoma by the clinical findings and course, because the treatment for this angiosarcoma was needed to start as soon as possible and visual examination was enough for the specialists to diagnose. Further therapy was conducted by them.

The dermatologists administered concurrent treatments of chemotherapy (weekly DTX; $25 \mathrm{mg} \cdot \mathrm{m}^{-2}$, six treatments, $258 \mathrm{mg}$ total) and radiotherapy (70 Gy), which led to a decrease in the cervical swelling and reduced the tumor to $47 \%$ of its original volume. Additional biweekly, outpatient treatment with DTX (15 treatments, $645 \mathrm{mg}$ total), caused the primary tumor and the cervical metastasis to disappear, although the scalp ulceration remained. A biopsy of the ulcer revealed no residual malignant cells. Resection of the ulcer lesion was performed and showed only the scar tissue. Six months later, the
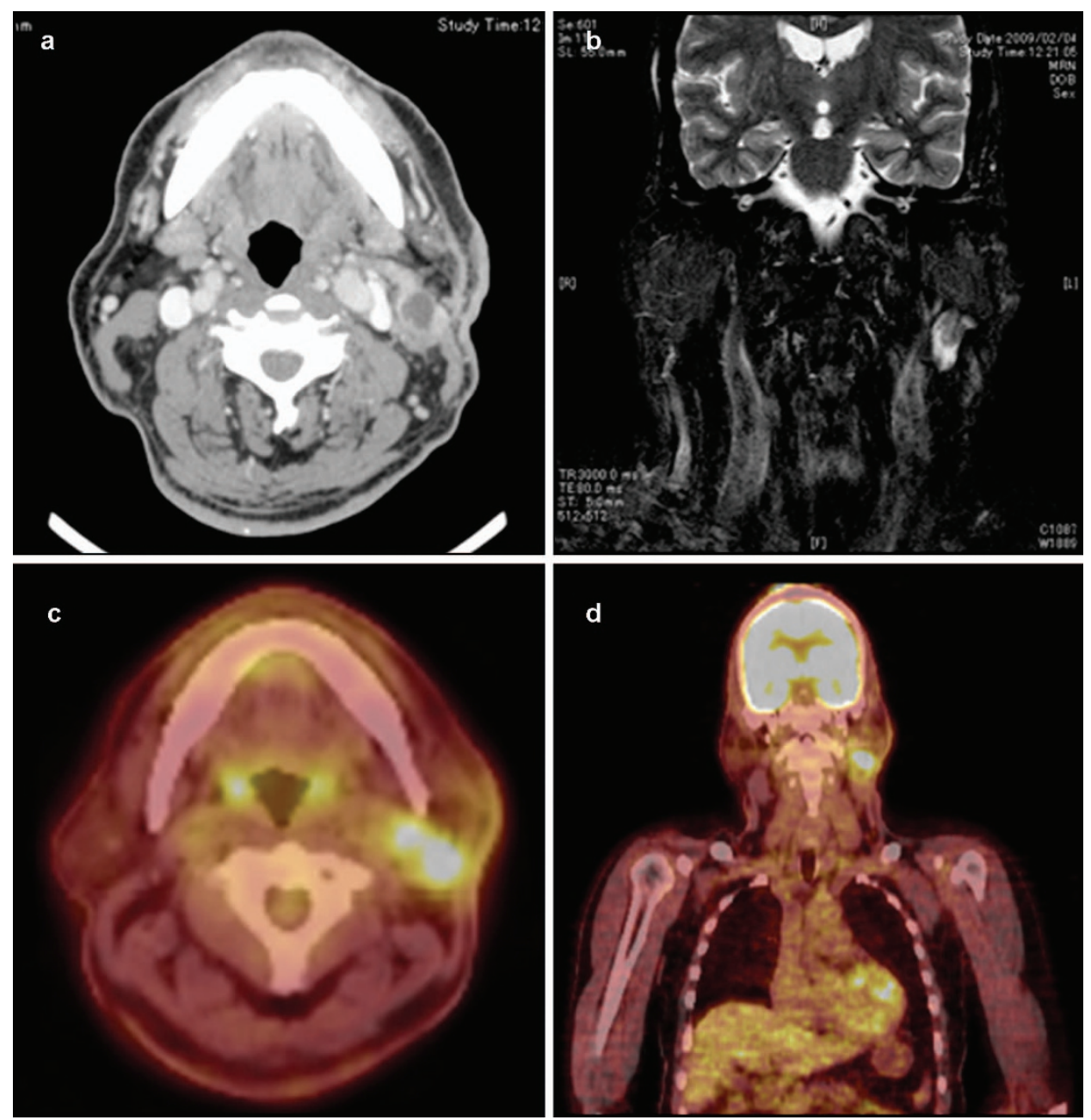

Figure 2 Radiological findings of the cervical lesion. (a) CT scanning image showing a round, swollen, ring-enhanced lymph node in the left cervical region. (b) MRI revealing a high-signal lesion on T2-weighted images under the left parotid gland. (c and d) PET CT scan image showing focal uptake in the left cervical region. (c) Axial view. (d) Whole-body view. CT, computed tomography; MRI, magnetic resonance image; PET, positron emission tomography. 

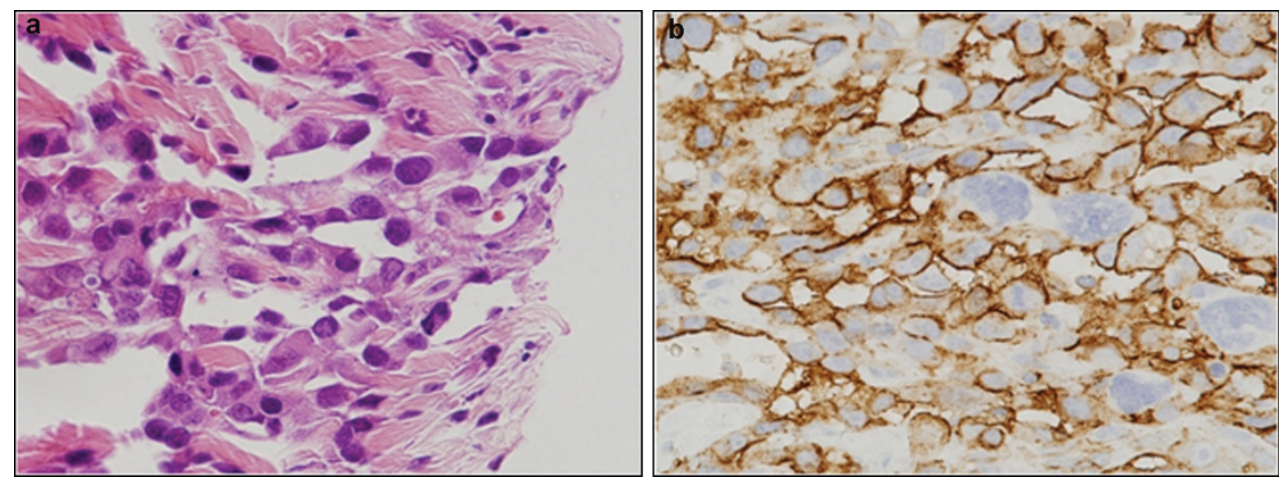

Figure 3 Histological and immunohistochemical aspects. (a) Histological features of the neck tumor showing enlarged pleomorphic endothelial cells and microcysts (hematoxylin and eosin staining, $\times 400)$. (b) Immunohistochemical staining showing CD31-positive tumor cells $(\times 400)$.

lymph node metastasis in front of the left auricle reappeared. Radiotherapy (70 Gy) was performed to treat the preauricular lymph node metastasis, followed by biweekly doxorubicin treatments $\left(20 \mathrm{mg} \cdot \mathrm{m}^{-2}\right.$, three times). Approximately 2 years after the final ulcer resection, the patient appears free of local recurrence and lung metastasis.

\section{DISCUSSION}

Angiosarcoma is a rare malignant tumor derived from vascular endothelial cells that usually follows an aggressive course, marked by local invasion and early lung metastasis. ${ }^{3,5}$ The occurrence of this neoplasm in the oral cavity is extremely rare. ${ }^{1,6-7} \mathrm{We}$ describe a patient with an angiosarcoma of the scalp that was detected initially by a patient's complaint of neck swelling and tenderness. Although reports indicate that cancers of unknown primary origin can be diagnosed by cervical lymphnode metastasis, ${ }^{8-10}$ this is the first case report in which angiosarcoma of the scalp was diagnosed in this manner.

Although patients generally describe pain and swelling at the primary lesion site, this patient complained only of painful neck inflammation, without any indication of pain or swelling of the scalp. Imaging procedures such as CT, MRI and US were not sufficient to diagnose this case. Ordinary in our institution, the vertex site is out of area in scanning CT or MRI for neck lesions. A retrospective examination of the PET CT scan indicated an uptake in the vertex of the head, but the scalp lesion was barely visible (Figure $2 \mathrm{~d}$ ). In actually radiologists did neither detect the primary lesion. It will be important that we have to consider a possibility of an existence of the vertex lesion in such case. The whole-body PET CT indicated a left cervical lymphnode metastasis. More careful observation and physical examination was necessary in this case to identify the primary cancer. A revised diagnostic protocol should note that cervical lymph node metastasis of unknown primary origin may result from angiosarcoma of the scalp. In addition, an echo-guided needle biopsy was an effective way to diagnose the lymph node metastasis, which has been reported for other angiosarcomas as well. ${ }^{11}$

Patient prognosis for angiosarcoma indicates a median survival rate of 16 months and cumulative survival rates at 2 and 5 years of $31 \%$ and $8 \%$, respectively. ${ }^{12}$ Although the optimal treatment remains uncertain, a few reports indicate an extended period of remission or complete remission following sequential weekly and monthly DTX treatments. ${ }^{3,13-14}$ Here we report that the concurrent chemoradiotherapy (weekly DTX and radiotherapy) and biweekly DTX in outpatient clinic was effective. Over half of angiosarcoma patients ultimately develop metastases, particularly to the lung. ${ }^{15}$ However, in the present case report, there is no evidence of recurrence 3 years

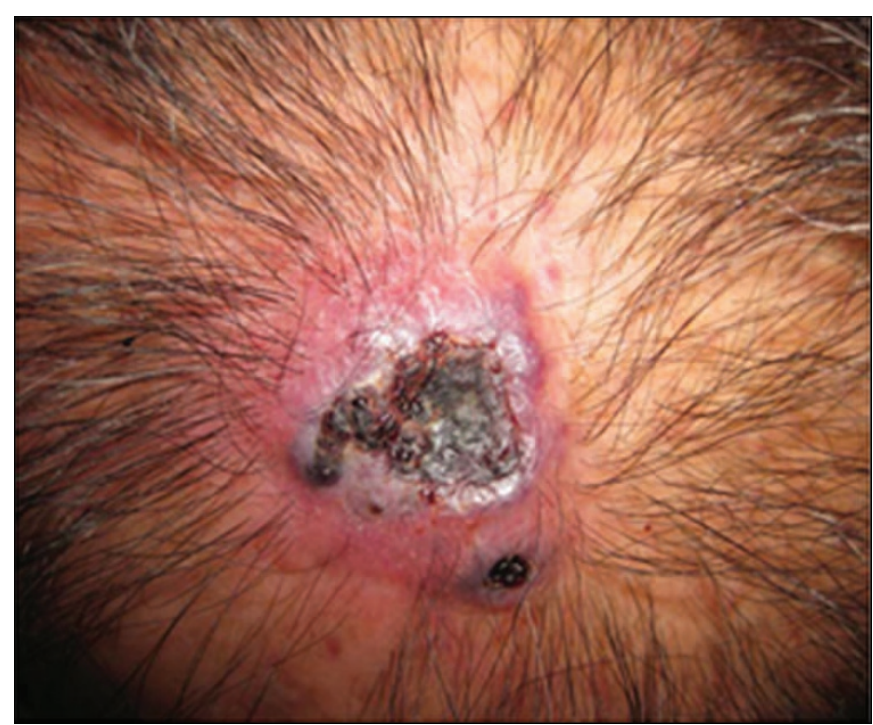

Figure 4 Clinical appearance of the vertex of head.

after the first medical examination. Thus, DTX prove to be an effective first-line therapy for angiosarcoma.

1 Arribas-Garcia I, Dominguez MF, Alcala-Galiano A et al. Oral primary angiosarcoma of the lower lip mucosa: report of a case in a 15-year-old boy. Head Neck 2008; 30(10): 1384-1388.

2 Guadagnolo BA, Zagars GK, Araujo D et al. Outcomes after definitive treatment for cutaneous angiosarcoma of the face and scalp. Head Neck 2011; 33(5): 661-667.

3 Nakamura Y, Hori E, Furuta J et al. Complete long-term response of angiosarcoma of the scalp with cervical lymph node metastases treated with a combination of weekly and monthly docetaxel. Br J Dermatol 2010; 163(6): 1357-13578.

4 Terada T. Fatal poorly differentiated angiosarcoma of the scalp. Int J Clin Exp Pathol 2010; 3(5): 541-544

5 Pawlik TM, Paulino AF, McGinn CJ et al. Cutaneous angiosarcoma of the scalp: a multidisciplinary approach. Cancer 2003; 98(8): 1716-1726.

6 Uchiyama $\mathrm{Y}$, Murakami S, Kishino $\mathrm{M}$ et al. A case report of primary gingival angiosarcoma. Oral Surg Oral Med Oral Pathol Oral Radiol Endod 2009; 108(6): e17-e21.

7 Terada T. Angiosarcoma of the oral cavity. Head Neck Pathol 2011; 5(1): 67-70.

8 Cerezo L, Raboso E, Ballesteros AI. Unknown primary cancer of the head and neck: a multidisciplinary approach. Clin Trans/ Oncol 2011; 13(2): 88-97.

9 Nassenstein K, Veit-Haibach P, Stergar $\mathrm{H}$ et al. Cervical lymph node metastases of unknown origin: primary tumor detection with whole-body positron emission tomography/computed tomography. Acta Radio/ 2007; 48(10): 1101-1108. 
10 Paul SA, Stoeckli SJ, von Schulthess GK et al. FDG PET and PET/CT for the detection of the primary tumour in patients with cervical non-squamous cell carcinoma metastasis of an unknown primary. Eur Arch Otorhinolaryngol 2007; 264(2): 189 195.

11 Pohar-Marinsek Z, Lamovec J. Angiosarcoma in FNA smears: diagnostic accuracy, morphology, immunocytochemistry and differential diagnoses. Cytopathology 2010 21(5): 311-319.

12 Itakura $\mathrm{E}$, Yamamoto $\mathrm{H}$, Oda $\mathrm{Y}$ et al. Detection and characterization of vascular endothelial growth factors and their receptors in a series of angiosarcomas. J Surg Oncol 2008; 97(1): 74-81.

13 Nagano T, Yamada Y, Ikeda T et al. Docetaxel: a therapeutic option in the treatment of cutaneous angiosarcoma: report of 9 patients. Cancer 2007; 110(3): 648-651.
14 Penel N, Lansiaux A, Adenis A. Angiosarcomas and taxanes. Curr Treat Options Oncol 2007; 8(6): 428-434.

15 Abraham JA, Hornicek FJ, Kaufman AM et al. Treatment and outcome of 82 patients with angiosarcoma. Ann Surg Oncol 2007; 14(6): 1953-1967.

(c) This work is licensed under a Creative Commons

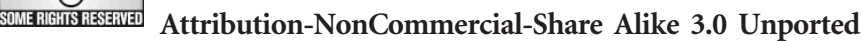
License. To view a copy of this license, visit http://creativecommons. org/licenses/by-nc-sa/3.0 